\title{
AlN Based RF MEMS Tunable Capacitor with Air- Suspended Electrode with Two Stages
}

\author{
Seong J. Cheon, Woo J. Jang, Hyeon S. Park, Min K. Yoon, and Jae Y. Park
}

\begin{abstract}
In this paper, a MEMS tunable capacitor was successfully designed and fabricated using an aluminum nitride film and a gold suspended membrane with two air gap structure for commercial RF applications. Unlike conventional two-parallelplate tunable capacitors, the proposed tunable capacitor consists of one air suspended top electrode and two fixed bottom electrodes. One fixed and the top movable electrodes form a variable capacitor, while the other one provides necessary electrostatic actuation. The fabricated tunable capacitor exhibited a capacitance tuning range of $375 \%$ at $2 \mathrm{GHz}$, exceeding the theoretical limit of conventional twoparallel-plate tunable capacitors. In case of the contact state, the maximal quality factor was approximately 25 at $1.5 \mathrm{GHz}$. The developed fabrication process is also compatible with the existing standard IC (integrated circuit) technology, which makes it suitable for on chip intelligent transceivers and radios.
\end{abstract}

Index Terms - Tunable capacitor, RF MEMS, aluminum nitride, air-gap, wide tuning range, capacitance

\section{INTRODUCTION}

A Micro electro mechanical systems (MEMS) technology enabling the batch fabrication of miniaturized

Manuscript received Apr. 28, 2012; revised Oct. 30, 2012. S. Cheon, Woo J. Jang, H. Park, M. Yoon, and J. Park are with Micro/Nano Devices and Packaging Laboratory, Department of Electronic Engineering, Kwangwoon Univ., 447-1 Wolgye-Dong, Nowon-Gu, Seoul, 139-701, Korea.

E-mail : jaepark@kw.ac.kr mechanical structures, devices, and systems has been originated from the Integrated circuit (IC) technology [1]. The MEMS technology has become more important for the development of radio frequency (RF) and microwave communication systems since it can be used to implement the free-standing or movable threedimensional (3-D) microstructures and hence achieve higher quality-factor (Q-factor) than many conventional IC devices. RF MEMS components that are currently under development in laboratories and industries around the world include switches, tunable capacitors, MEMS inductor with high Q factor [2], film bulk acoustic resonators (FBARs), and MEMS resonators and filters [3-5].

For instance, various MEMS based tunable capacitors have been applied in the RF front-end building blocks of the modern RF/microwave communication systems, such as voltage controlled oscillators (VCOs) [6-8], tunable filters [9-13], and impedance matching networks [14]. These MEMS tunable capacitors should have widetuning range [15], high Q-factor [16] and low driving voltage [17] for being successfully commercialized. Among all the micromachined tunable capacitors developed to date, the parallel-plate configuration using electrostatic actuation is most commonly used. The parallel-plate capacitor can be fabricated using surface micromachining processes established well. However, the tuning range of such capacitors is limited to $50 \%$ by the pull-in effect. The pull-in instability, due to which only $33 \%$ of the gap between plates could be covered smoothly, greatly reduces the useful dynamic range of MEMS tunable capacitors.

In this paper, a RF MEMS tunable capacitor is newly designed and fabricated by using aluminum nitride 
dielectric film and a movable electrode with two different air gap structures. This design keeps the simplicity of conventional two-parallel-plate configuration, while overcoming its low tuning range. In addition, an aluminum nitride film is used as a high dielectric layer in order to increase a tuning range, decrease an operating voltage, and be integrated with FBAR (Film Bulk Acoustic Resonator) device for on chip tunable filters and intelligent radios.

\section{DESIGN}

Fig. 1 shows a 3-dimensional geometrical structure of the proposed MEMS tunable capacitor. The proposed tunable capacitor has the structure of 2-port network using a shunt capacitor with air-suspended metal and fixed signal electrodes. As shown in Fig. 2, the conventional parallel-plate variable capacitor with single gap-spacing, that is $g_{1}$ or $g_{2}$, and its maximum tuning range is theoretically limited to $50 \%$. To substantially enhance the maximum tuning range of the tunable

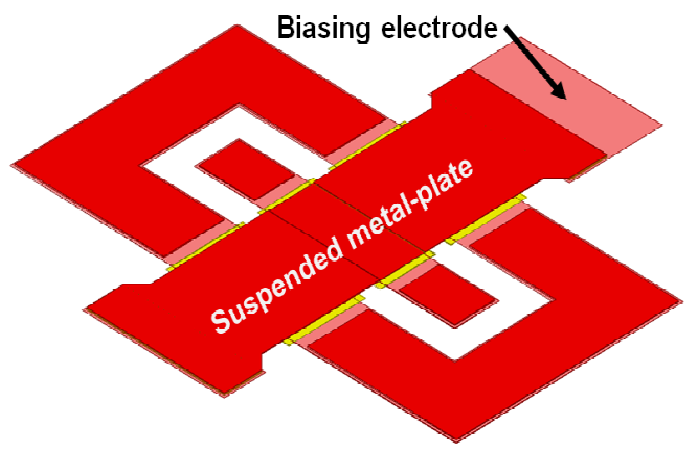

Fig. 1. 3-D geometrical structure of the proposed tunable RF MEMS capacitor with an air-suspended movable electrode with two stages.

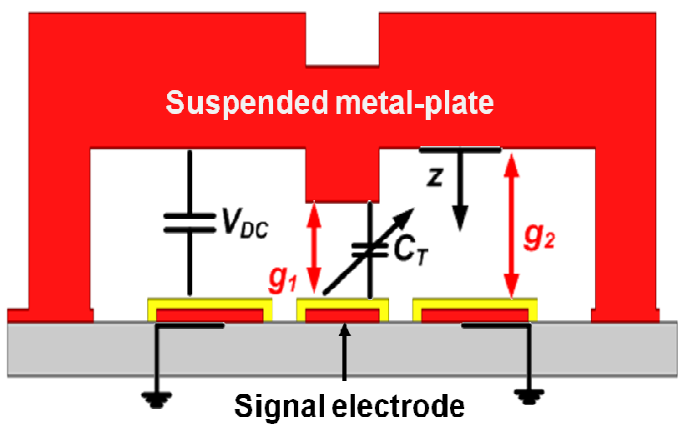

Fig. 2. A cross-sectional drawing of the proposed tunable capacitor with an air-suspended movable electrode with two different stages. capacitor, two air gap structure was adopted with one-tothree ratio of the $g_{1}$ ranging from 1 to $2 \mu \mathrm{m}$ and $g_{2}$ with 3 $\mu \mathrm{m}$. The structure with two air gap was simply implemented using the double exposure process of photolithography and electroplating technique. At first, the area of air-suspended metal electrode with the distance of $g_{l}$ was contacted onto the fixed signal electrode due to the electrostatic force by $V_{D C}$. Moreover, in this case, the capacitance of the proposed tunable capacitor was smoothly increased, because the height of $g_{1}$ was lower than a third height of $\mathrm{g} 2$, which is the height by pull-in voltage.

In an initial state, the capacitance could be obtained by using an effective dielectric constant, $\varepsilon_{\text {eff }}[14]$

$$
\sqrt{\varepsilon_{e f f}}=\left[1+\left(a_{1}-b_{1} \ln \left(\frac{w}{b}\right)\right)\left(\frac{1}{\sqrt{\varepsilon_{r}}}-1\right)\right]^{-1}
$$

where $\mathrm{W}$ is the width of the conductor line and $\varepsilon_{r}$ is the relative dielectric constant of the aluminum nitride film in the designed MEMS tunable capacitor. The coefficients $a_{1}$ and $b_{1}$ are calculated by the relationship between $a$ of the thickness of silicon substrate and $b$ of the air gap. The coefficients $a_{1}$ and $b_{1}$ are specified by

$$
\begin{gathered}
a_{1}=0.155+0.505\left(\frac{a}{b}\right) \\
b_{1}=0.023+0.1863\left(\frac{a}{b}\right)-0.194\left(\frac{a}{b}\right)^{2}
\end{gathered}
$$

In the initial state, the calculated capacitance of the designed MEMS tunable capacitor was $0.217 \mathrm{pF}$ by using the above equations. In the contact state, the calculated capacitance was $0.593 \mathrm{pF}$ by using the measured relative dielectric constant of 8.2.

Fig. 3 shows the 3-D EM simulated capacitance results of the proposed tunable capacitor at an initial and a contact states. As shown in Fig. 3, the proposed tunable capacitor without any bias voltages had the capacitance about $0.278 \mathrm{pF}$. After being contacted by applying the operation voltage, the tunable capacitor exhibited the capacitance of approximately $0.78 \mathrm{pF}$ due to the fringing capacitance occurred between two electrodes.

Another important characteristic parameter of the MEMS tunable capacitor is the $Q$-factor. For the 


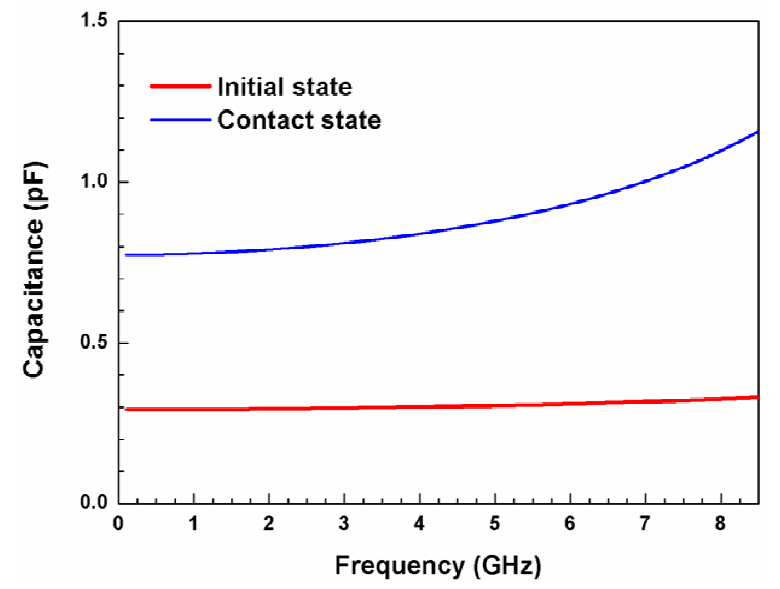

Fig. 3. 3-D EM simulated capacitances vs. frequency of the proposed RF MEMS tunable capacitor at initial and contact states.

improvement of the $Q$-factor, this study adopted a high resistivity silicon substrate $(2 \mathrm{k}$ to $10 \mathrm{k} \Omega-\mathrm{cm})$ to reduce effectively the RF loss occurred form the substrate.

\section{FABRICATION}

Fig. 4 shows the detailed fabrication sequences of the proposed RF MEMS tunable capacitor with an airsuspended movable electrode with two stages. The proposed MEMS tunable capacitor was fabricated on high resistivity silicon substrate (2- to $10-\mathrm{k} \Omega-\mathrm{cm}$ ) of 525 $\mu \mathrm{m}$ in thickness for reducing the substrate loss. After depositing silicon oxide film as an insulator on the substrate, bottom electrodes of platinum and aluminum nitride film were deposited and patterned in sequence with the thickness of $0.35 \mu \mathrm{m}$ and $0.2 \mu \mathrm{m}$, respectively. As shown in Fig. 4(c), AZ7220 photoresist with the thickness of $3 \mu \mathrm{m}$ was then double-coated and patterned as a sacrificial layer for forming the air-suspended movable electrode by double expose etch technique. A gold material with the thickness of $0.2 \mu \mathrm{m}$ for seed layer and a Ti material with the thickness of $500 \AA$ for adhesion layer were then sputtered on the top of the patterned sacrificial layer. After forming the thick photoresist molds for electroplating process, $\mathrm{Au}$ was electroplated to form thick air-suspended movable electrode with the thickness of $4 \mu \mathrm{m}$ for improvement of reliability of RF MEMS tunable capacitors. Finally, after making etch holes on the thin gold seed layer, the sacrificial layer was finally removed by using acetone

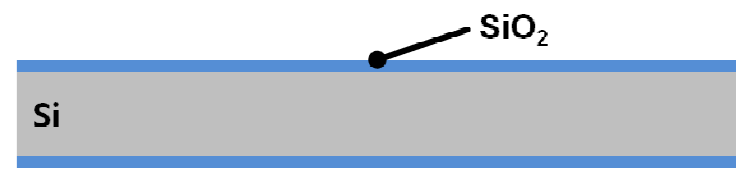

(a)

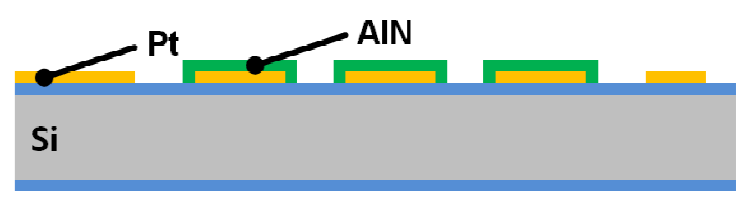

(b)

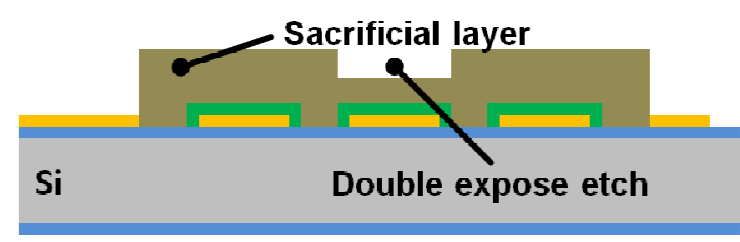

(c)

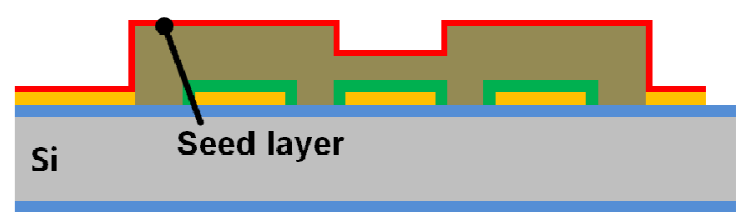

(d)

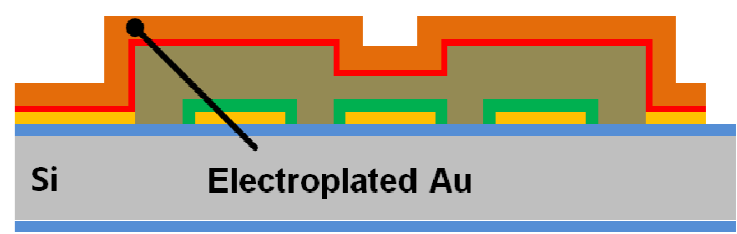

(e)

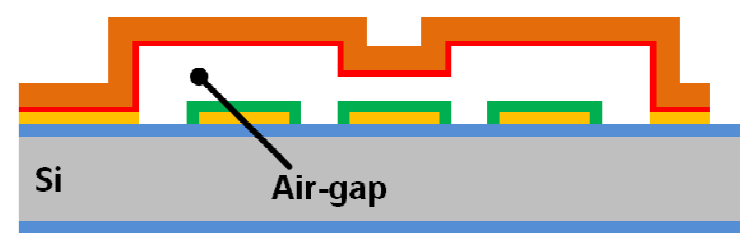

(f)

Fig. 4. Fabrication sequences of the proposed RF MEMS tunable capacitor with an air-suspended movable electrode with two stages.

and critical point dryer (CPD) process.

Fig. 5(a) and (b) shows a scanning electron microscope (SEM) photograph and a close-up view of the fabricated RF MEMS tunable capacitor. The formed height between $\mathrm{SiO}_{2}$ layer and movable metal-plate was approximately $3 \mu \mathrm{m}$. Moreover, the formed height between AlN dielectric material and movable metal-plate 


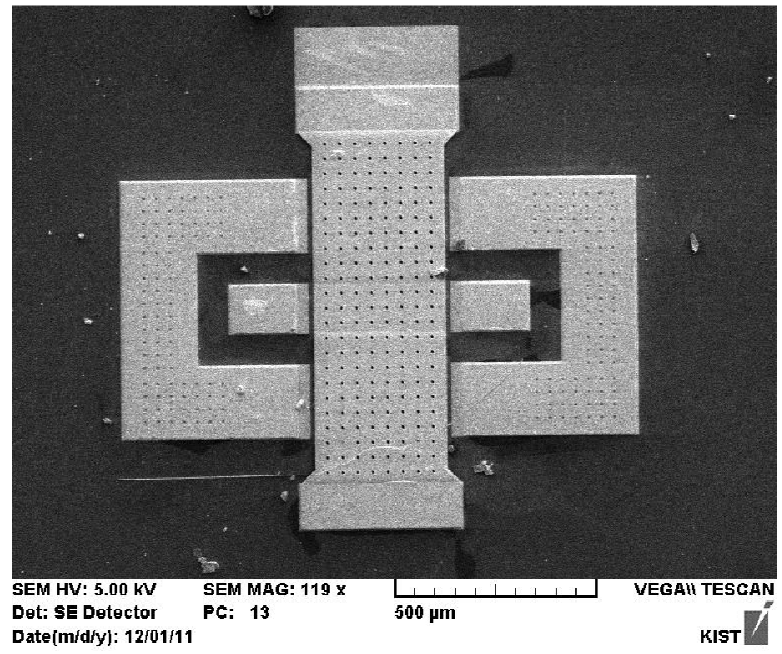

(a)

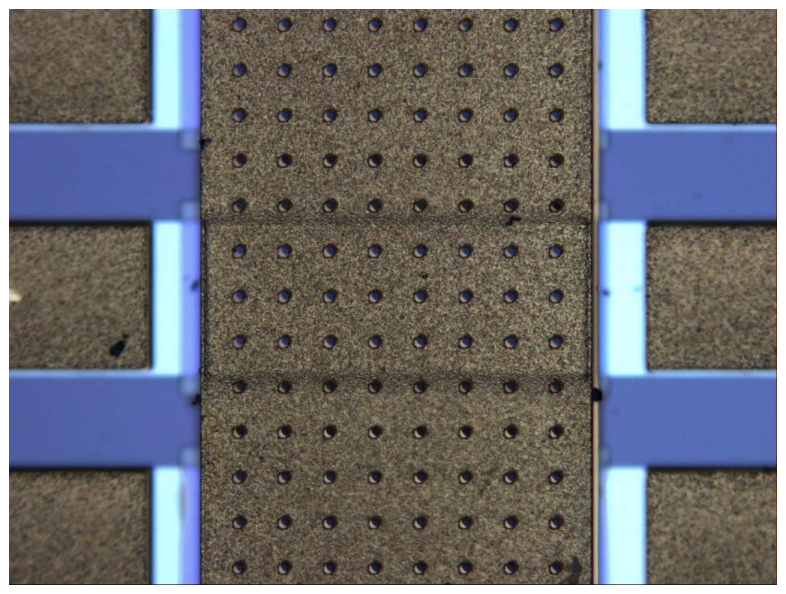

(b)

Fig. 5. A SEM photograph (a) and its close-up view, (b) of the fabricated RF MEMS tunable capacitor with an air-suspended movable electrode with two stages.

by double-exposed etching was approximately $1.95 \mu \mathrm{m}$. Its size was $1.2 \mathrm{~mm} \times 1.2 \mathrm{~mm}$. The contact area between the air-suspended movable electrode and the fixed signal electrode was $120 \mu \mathrm{m} \times 300 \mu \mathrm{m}$. Moreover, each bias area between the movable electrode and the fixed ground plane was $180 \mu \mathrm{m} \times 300 \mu \mathrm{m}$.

\section{MEASUREMENT RESUlTS}

The fabricated MEMS tunable capacitor was measured and characterized by using an Agilent E5071 network analyzer and PICOPROBE GSG with $250 \mu \mathrm{m}$ pitch size. A short-open-load-through (SOLT) has been adopted to calibrate the characteristics of the measurement equipment before measuring the fabricated capacitors.
The measured frequencies were ranged from 1 to $8 \mathrm{GHz}$ for commercial RF and microwave applications. The applied actuation voltage was ranging from 0 to $75 \mathrm{~V}$.

Before fabricating the proposed tunable capacitor, the MIM capacitors with the aluminum nitride film were first fabricated, measured, and characterized. Fig. 6 shows the capacitance and the quality factor behaviors of the capacitor with the area of $80 \mu \mathrm{m} \times 80 \mu \mathrm{m}$ at commercial radio frequencies. The maximal quality factor was higher than 30 and the capacitance density was $270 \mathrm{pF} / \mathrm{mm}^{2}$. The measured dielectric constant and dielectric loss of the fabricated aluminum nitride were 8.2 and 0.01 , respectively.

Fig. 7 shows the measured capacitances of the fabricated MEMS tunable capacitor at the frequency of $2 \mathrm{GHz}$. When the operation voltage was not applied, the fabricated capacitor had the capacitance of $0.317 \mathrm{pF}$, as

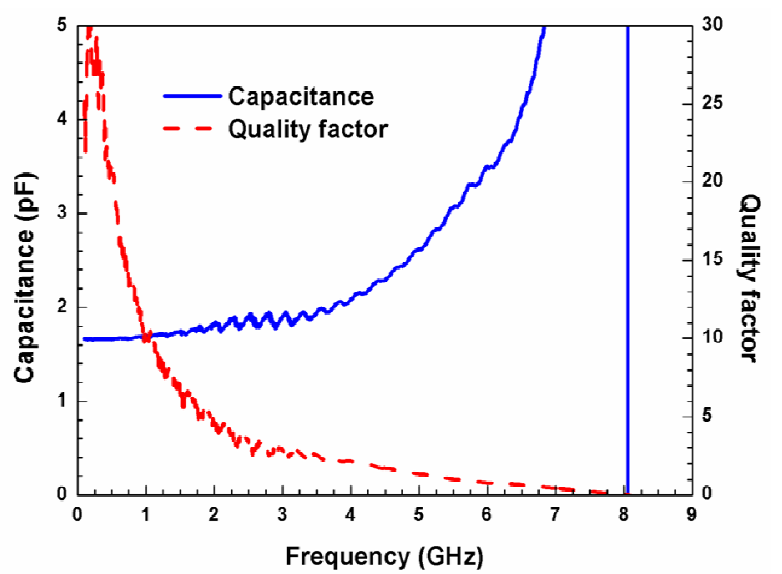

Fig. 6. Measured capacitance and quality factor of the fabricated AlN based MIM capacitor.

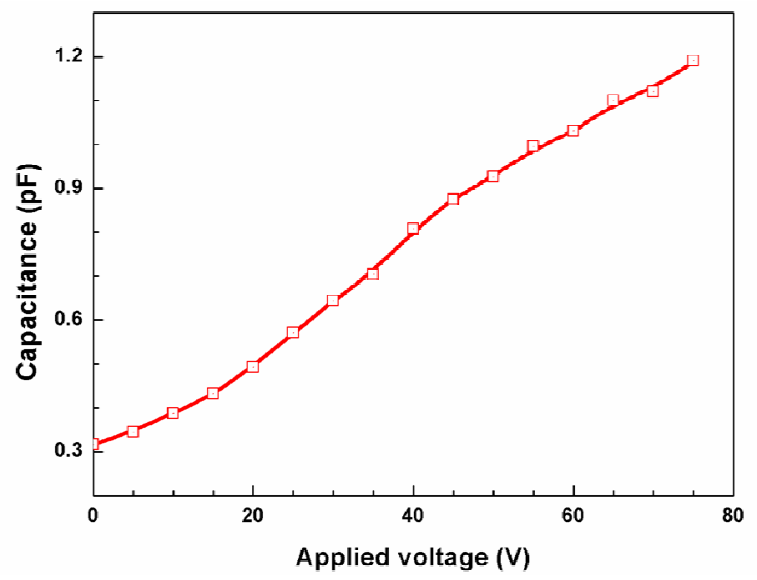

Fig. 7. Measured capacitances of the fabricated RF MEMS tunable capacitor at various applied voltages. 


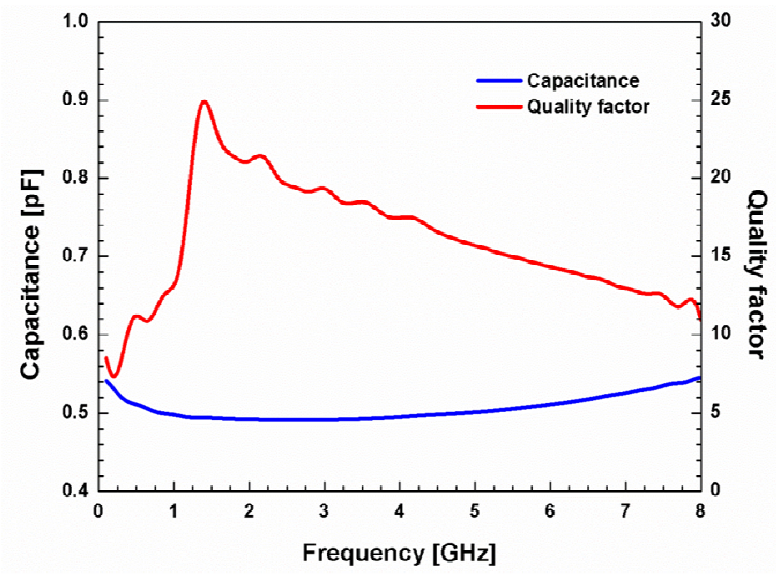

Fig. 8. Measured capacitance and quality factor of the fabricated RF MEMS tunable capacitor at the applied voltage of $30 \mathrm{~V}$.

expected through the EM simulation. In the contact state, the capacitance was approximately $0.62 \mathrm{pF}$. The airsuspended movable electrode was contacted on the fixed signal electrode at the applied voltage of $30 \mathrm{~V}$. It was slightly lower than the EM simulated capacitance of $0.78 \mathrm{pF}$. This discrepancy might be caused by the enlarged etch holes compared with the designed values. As shown in Fig. 7, the capacitance was linearly increased at the applied voltages higher than $30 \mathrm{~V}$ due to the zipping effects. The tunable capacitor exhibited a capacitance of $1.19 \mathrm{pF}$ and a capacitance tuning range of $375 \%$ at the applied voltage of $75 \mathrm{~V}$, which make it suitable for tunable filter and antenna applications. Fig. 8 shows the measured capacitance and quality factor of the MEMS tunable capacitor. In the contact state, the maximal quality factor of 25 was obtained at $1.5 \mathrm{GHz}$.

\section{CONCLuSions}

In this paper, a MEMS tunable capacitor with wide tuning range was successfully designed and fabricated by using aluminum nitride film and a gold suspended membrane with two air gap structures for commercial RF applications. The utilized high dielectric aluminum nitride film and two air gap structured membrane were effective to achieve a wide-tuning range and high capacitance density of the MEMS tunable capacitor, as expected. In addition, the developed fabrication sequence was simplified and compatible with the aluminum nitride based FBAR devices resulting in on chip tunable filters and radios with high performances and small size/volumes.

\section{ACKNOWLEDGMENTS}

This research was supported by the Intelligent RF Engineering Research Center (ERC) of the Korea Ministry (Grant No. R11-2005-029-06004-0) of Science and Technology. The authors are grateful to the MiNDaP group members for their technical supports and discussions.

\section{REFERENCES}

[1] Jae Y. Park, et al, "RF MEMS Devices for Wireless Applications", Journal of Semiconductor Technology and Science, Vol.1, No.1, pp.70-83, Mar., 2001.

[2] Seonho Seok, et al, "A High Performance Solenoid-Type MEMS Inductor", Journal of Semiconductor Technology and Science, Vol.1, No.3, pp.1-7, Sep., 2001.

[3] A. Q. Liu, et al, "RF MEMS Switches and Integrated Wsitching Circuits", Journal of Semiconductor Technology and Science, Vol.7, No.3, pp.166-176, Sep., 2007.

[4] Harrie A C Tilmans, et al, "MEMS for Wireless Communications: 'from RF-MEMScomponents to RF-MEMS SiP'”, Journal of Micronechanics and Microengineering, Vol.13, No.4, pp.139-163, Jun., 2003.

[5] Jong-Man Kim, et al, "A Micromachined Two-state Bandpass Filter using Series Inductors and MEMS Switches for WLAN Applications", Journal of Micronechanics and Microengineering, Vol.4, No.4, pp.300-306, Dec., 2004.

[6] Aleksander Dec and Ken Suyama, "Microwave MEMS-Based Voltage-Controlled Oscillators", IEEE Transactions on Microwave Theory and Techniques, Vol.48, No.11, pp.1943-1949, Nov, 2000.

[7] Charles L. Goldsmith, et al, "RF MEMS Variable Capacitos for Tunable Filters", International Journal of RF and Microwave Computer and Aided Engineering, Vol.9, No.4, pp.362-374, Jul., 1999.

[8] Maneesha Yellepeddi and Kartikeya Mayaram, "Issues in the Design and Simulation of a MEMS VCO based Phase-Locked Loop", IEEE 
International Symposium on Circuits and Systems 2007, 27-30, May 2007, New Orleans, US, pp.1553-1556.

[9] A. Abbaspour-Tamijani, et al, "Miniature and Tunable Filters using MEMS Capacitors", IEEE Transactions on Microwave Theory and Techniques, Vol.51, No.7, pp.1878-1885, Jul., 2003.

[10] S. Pipilos, et al, "A Si $1.8 \mathrm{GHz}$ RLC filter with tunable center frequency and quality factor", SolidState Circuit, IEEE Journal of, Vol.31, No.10, pp.1517-1525, Oct., 1996.

[11] Naibo Zhang, et al, "Design and Analysis of a Tunable Bandpass Filter Employing RF MEMS Capacitors", Electron Device Letters, IEEE, Vol.32, No.10, pp.1460-1462, Oct., 2011.

[12] Kamran Entesari, et al, "A 25-75-MHz RF MEMS Tunable Filter", IEEE Transactions on Microwave Theory and Techniques, Vol.55, No.11, pp.23992405, Nov, 2007.

[13] Montserrat Fernandez-Bolanos, et al, "Centre frequency and bandwidth tunable bandpass filter based on RF MEMS (10-14GHz)", Microwave Conference, 2009. EuMC 2009. European, Sep. 29 - Oct. 1, 2009, Rome, Italy, pp.1708-1711.

[14] Arda Deniz Yalqnkaya, et al, "High aspect ratio MEMS Capacitor for high frequency impedance matching applications", Proceedings of the $10^{\text {th }}$ IEEE International Conference on Electronics, Circuits and Systems, ICECS,14-17 Dec. 2003, Sharjah, UAE, Vol.2, pp.918-912.

[15] Darrin J. Young, et al, “A Low-Noise RF VoltageControlled Oscillator Using On-Chip High-Q Three-Demensional Coil Inductor and Micromachined Variable Capacitor", Solid-State Sensors and Actuator Workshop, 8-11, June 1998, Hilton Head Island, Scottleland, pp.128-131.

[16] Maher Bakri-Kassem, and Raafat R. Mansour, "Two movable-plate nitride-loaded MEMS variable capacitor", IEEE Transactions on Microwave Theory and Techniques, Vol.52, No.3, pp.831-837, Mar., 2004.

[17] Darrin J. Young, et al, “A Low-Noise RF VoltageControlled Oscillator Using On-Chip High-Q Three-Demensional Coil Inductor and Micromachined Variable Capacitor", Solid-State Sensors and Actuator Workshop, 8-11, June 1998, Hilton Head Island, Scottleland, pp.128-131.
[18] J. Helszajn, Microwave Planar Passive Circuits and Filters, pp.2-17, England, John Wiley \& Sons Ltd., 1994..

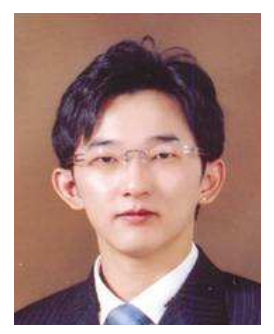

Seong J. Cheon received B.S. and M.S. degrees in the Electronic Engineering from Kwangwoon University, Seoul, Korea, in 2006 and 2008, respectively. $\mathrm{He}$ is currently working toward a Ph. D. degree in the Department of Electronic Engineering from Kwangwoon University. His current research interests are in the areas of RF microelectromechanical systems (MEMS) actuators, microelectronic devices, RF front-end modules, embedded passive devices (EPDs), and system on packaging (SOP).

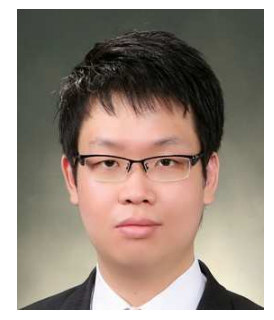

Woo J. Jang received the B.S. and M.S. degree in the Electronic Engineering from Kwangwoon University, Seoul, Korea, in 2010 and 2012, respectively. $\mathrm{He}$ is currently working at Research and Division in Samsung Electronics Co., Ltd. His current research interests are RF MEMS switches and tunable filters.

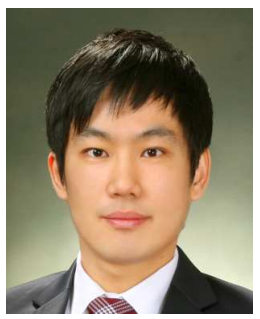

Hyeon S. Park received the B.S. degree in the Electronic Engineering from Kwangwoon University, Seoul, Korea, in 2012. He is currently working toward the M.S. degree in the Electronic Engineering at Kwangwoon University. His current research interests are RF MEMS switches and tunable filters. 


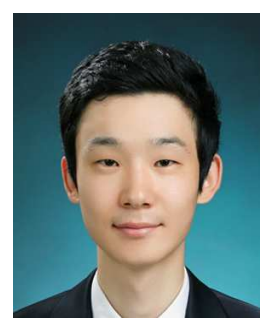

Min K. Yoon received B.S. degree in the Dept. of Electronic Engineering from Kwangwoon University, Seoul, Korea, in 2012. He has been working toward the M.S. degree in MEMS based devices in the Department of Electronic engineering from Kwangwoon University. His research focuses on various MEMS actuators, such as electro static MEMS switches, tunable devices and RF passive devices.

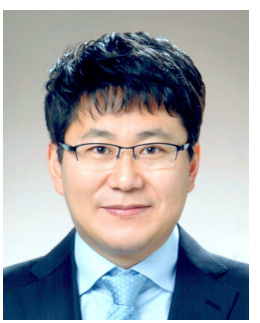

Jae Y. Park received the M.S.E.E. and Ph.D. degree in electrical and computer engineering from Georgia Institute of Technology, Atlanta, in 1995 and 1997, respectively. $\mathrm{He}$ worked at the Packaging Research Center, Georgia Institute of Technology as a Research Engineering for two years. He has also worked at the Micro-System Group in the LG Electronics Institute of Technology as a Team Leader of RF MEMS Research for six years. In September 2004, he joined the faculty of the Department of Electronics Engineering, Kwangwoon University, Seoul, Korea. He has published more than 170 journal articles and conference proceedings and field more than 95 patents. His current research interests include microelectromechanical systems (MEMS, micro-actuators for optical and RF applications), micro-system packaging (wafer level packaging, SOP, SOC, multichip modules), micro/nano electro-chemical sensors, smart dust, and RF MEMS components. 